\title{
The Role of American Thyroid Association Pediatric Thyroid Cancer Risk Stratification and BRAFV600E Mutation in Predicting the Response to Treatment in Papillary Thyroid Cancer Patients $\leq 18$ Years Old
}

\author{
(1) Yasemin Giles Şenyürek ${ }^{1}$, (1) Yalın İşcan ${ }^{1}$, (1) Issmail Cem Sormaz ${ }^{1}$, (1) Şükran Poyrazoğlu², (1) Fatih Tunca1 \\ 1 istanbul University, Istanbul Faculty of Medicine, Department of Surgery, Istanbul, Turkey \\ 2istanbul University, İstanbul Faculty of Medicine, Department of Pediatrics, Unit of Pediatric Endocrinology, Istanbul, Turkey
}

\section{What is already known on this topic?}

American Thyroid Association (ATA) pediatric initial risk stratification was documented to be useful and effective in predicting recurrence and response to treatment in papillary thyroid cancer (PTC). BRAF ${ }^{V 000 E}$ mutation has been found to be associated with an increased risk of lymph node metastasis, recurrence, and poor prognosis in adult patients. There is limited data about the impact of $B R A F^{V 600 E}$ mutation on prognosis in pediatric PTC.

\section{What this study adds?}

This study documented that ATA initial pediatric risk stratification effectively predicted the risk of recurrent and persistent disease and final response to treatment in PTC patients $\leq 18$ years old. The presence of $B R A F^{V 600 E}$ mutation was highly predictive for locoregional recurrence but had no significant effect on the final rate of excellent response to treatment.

\section{Abstract}

Objective: This study aimed to evaluate the role of risk stratification by the American Thyroid Association (ATA) pediatric thyroid cancer risk levels and $B R A F^{V 600 E}$ mutation to predict the response to treatment in papillary thyroid cancer (PTC) patients $\leq 18$ years old.

Methods: Clinical outcomes during a median period of 6 (2-21.8) years were assessed in 70 patients, according to ATA pediatric risk stratification, $B R A F^{V 600 E}$ mutation status, and dynamic risk stratification (DRS) at final follow-up.

Results: Of 70 patients, 44 (63\%), 14 (20\%), and 12 (17\%) were classified initially as low-, intermediate-, and high-risk, respectively. $B R A F^{V 600 E}$ mutation analysis data was available in $55(78.6 \%)$ patients, of whom $18(32.7 \%)$ had the BRAF ${ }^{\mathrm{V} 600 \mathrm{E}}$ mutation. AcCording to the final DRS, 61 (87\%), two (3\%), six (9\%), and one (1\%) patients were classified as an excellent, incomplete biochemical, incomplete structural, and indeterminate response, respectively. All ATA low-risk patients showed excellent response to treatment, whereas the rate of excellent response was $65.4 \%$ in intermediate- and high-risk levels ( $p<0.001$ ). The rates of excellent response in $B R A F^{V 600 E}$ positive and negative patients were $83 \%$ and $92 \%$, respectively $(\mathrm{p}=0.339)$. The rate of locoregional recurrence was significantly higher in $B R A F^{V 600 E}$ positive vs negative patients $(33.3 \%$ vs $2.7 \%$ respectively, $\mathrm{p}=0.001)$.

Conclusion: ATA pediatric risk stratification is effective in predicting response to treatment in PTC patients $\leq 18$ years old. The presence of $B R A F^{V 600 E}$ mutation was highly predictive for recurrence but had no significant impact on the rate of excellent response to treatment at final follow-up.

Keywords: $B R A F^{V 600 E}$ mutation, dynamic risk stratification, pediatric thyroid cancer, thyroid cancer

Address for Correspondence: Yalın İşcan MD, İstanbul University, İstanbul Faculty of Medicine, Department of Surgery, İstanbul, Turkey

Phone: +90 5428049232 E-mail: yaliniscan@gmail.com ORCID: orcid.org/0000-0002-5576-9496

- Copyright 2022 by Turkish Society for Pediatric Endocrinology and Diabetes

The Journal of Clinical Research in Pediatric Endocrinology published by Galenos Publishing House.
Conflict of interest: None declared Received: 26.10 .2021 Accepted: 20.01.2022 


\section{Introduction}

Differentiated thyroid cancer (DTC) in pediatric and adolescent populations is uncommon, and constitutes approximately $2-4 \%$ of all pediatric malignancies $(1,2)$. However, global trends in the incidence of thyroid cancer in children and adolescents showed rapid increases between 1998-2002 and 2008-2012 in almost all countries (3). Thyroid cancer is the most common endocrine malignancy in the 0-19 year age group (4). Papillary thyroid cancer (PTC) constitutes almost $90 \%$ of all thyroid carcinoma in this age group (5). The initial presentation, clinical course, and mortality of DTC in pediatric patients exhibit differences compared to adult patients. The rates of lymph node involvement, distant metastasis, and recurrence are much higher in pediatric and adolescent patients compared to adults, but the mortality rate at 20 years is less than $1 \%(6,7)$. The use of the American Joint Committee on Cancer/International Union Against Cancer (AJCC/UICC) TNM staging system is recommended for patients with DTC to predict disease-related mortality $(8,9)$. The role of the AJCC/UICC TNM staging system to predict prognosis in pediatric DTC is limited due to the very low mortality rate (8). Considering the differences between adult and pediatric DTC, the American Thyroid Association (ATA) published Management Guidelines for Children with Thyroid Nodules and Differentiated Thyroid Cancer in 2015 (1). This guideline recommended initial postoperative stratification of pediatric PTC patients into low-, intermediate-, or high-risk levels to predict the patients at risk of persistent or recurrent cervical disease (1). ATA pediatric initial risk stratification was documented to be useful and effective in predicting recurrence in many studies $(10,11,12,13,14)$. The dynamic risk stratification system (DRS), which was suggested to assess the response to therapy in DTC, has been validated in adult patients and has also been evaluated in pediatric DTC patients in some studies (12-20).

$B R A F^{V 600 E}$ mutation has been found to be associated with an increased risk of lymph node metastasis, recurrence, and poor prognosis in adult patients $(21,22)$. BRAF ${ }^{V 600 E}$ mutation status was incorporated into the ATA 2015 Modified Risk Stratification System for adult DTC patients and continuous risk scale for the assessment of structural recurrence risk to assist clinicians in proper risk stratification when mutation status data were available (8). There is limited data about the impact of $B R A F^{V 600 E}$ mutation on prognosis in pediatric PTC $(23,24,25,26)$. There are some reasons for the limited data. The rate of $B R A F^{V 600 E}$ mutation is low in pediatric PTC compared to adults; it exhibits great differences according to the age of the patient and is very low in young patients $(21,27)$. There is only one study in the literature that evaluated the correlation between BRAF ${ }^{V 600 E}$ mutation status and ATA pediatric initial risk stratification. The authors detected no significant correlation between the BRAF V600E mutation status and ATA pediatric initial risk stratification (24). The impact of BRAFV600E mutation on response to treatment by DRS has not been previously investigated in pediatric PTC patients.

The aim of this study was to evaluate the role of ATA pediatric thyroid cancer risk stratification and BRAF ${ }^{V 600 E}$ mutation status to predict the response to treatment in pediatric and adolescent PTC patients.

\section{Methods}

A total of 119 patients $\leq 18$ years old underwent thyroid surgery in the Division of Endocrine Surgery of Istanbul Faculty of Medicine, Department of General Surgery between 1995 and 2020. Of these 119 patients, 85 (71.4\%) were treated for PTC. This retrospective study included 70 (82\%) of the 85 patients in whom all clinicopathological and follow-up data were available. BRAF ${ }^{V 600 E}$ mutation analysis was performed in $55(78.6 \%)$ of 70 patients. Preoperative evaluation included thyroid hormone assay, neck ultrasonography (US), fine-needle aspiration biopsy (FNAB) of suspicious nodules, and FNAB or FNABthyroglobulin (Tg) washout of suspicious lymph nodes. The extent of initial thyroidectomy was either lobectomy or total thyroidectomy. Modified radical neck dissection (MRND) and therapeutic central neck dissection (TCND) were performed in patients with proven lateral neck metastasis. TCND was also performed in patients without lateral neck metastasis but with pre- or intra-operative evidence of clinically involved central lymph nodes. Routine prophylactic central neck dissection (PCND) was performed after 2010 in our institution in pediatric DTC patients.

Postoperative management of the patients was accomplished with a multidisciplinary approach, including the departments of pediatric endocrinology, nuclear medicine, and endocrine surgery. Stimulated Tg assay (sTg) and neck US was done 4-6 weeks after surgery in all patients. The patients were initially stratified as low, intermediate-, and high-risk postoperatively, according to the ATA risk stratification system for pediatric and adolescent DTC patients. Postoperative radioactive iodine (RAI) treatment was performed in all ATA intermediateand high-risk patients. The decision to use RAI treatment in ATA low-risk patients was individualized according to the clinicopathological features and postoperative sTg and antiTg (anti-Tg) values. A whole-body scan (WBS) was obtained 1 week after RAI treatment. Thyroid-stimulating hormone 
(TSH) suppression treatment was given in all patients aiming to keep TSH levels lower than $0.1 \mathrm{mIU} / \mathrm{L}$.

Neck US, either stimulated or nonstimulated Tg and anti-Tg assay were repeated every 6-12 months according to the clinical course and initial risk stratification. WBS with 2-5 mCi I I11 was performed 12 months after RAI treatment with concurrent measurement of sTg and anti-Tg in patients who received RAI treatment. The trend of serum anti-Tg levels was evaluated to manage the follow-up strategy in patients with positive $\mathrm{Tg}$ autoantibodies (TgAb). In patients with either detectable/rising levels of nonstimulated Tg or rising/ persistently high anti-Tg levels, diagnostic WBS and/or contrast-enhanced computerized tomography of neck and chest were performed when neck US was negative.

\section{BRAF ${ }^{V 600 E}$ Mutation Analysis}

$B R A F^{V 600 E}$ mutation analysis was performed in formalinfixed, paraffin-embedded thyroid tissue of thyroid tumor specimens. The QIAamp DNA tissue kit (Qiagen, Hilden, Germany) was used for genomic DNA preparation, following the manufacturer's instructions. BRAF ${ }^{V 600 E}$ mutation was determined by pyrosequencing using the Qiagen PyroMark Q24 pyrosequencer (Qiagen, Venlo, Netherlands) according to the manufacturer's instructions, as has been reported previously (28).

\section{Definitions of Recurrence and Response to Treatment}

In patients who underwent total thyroidectomy, with or without RAI treatment, a disease-free state was defined as a nonstimulated Tg level $<0.2 \mathrm{ng} / \mathrm{mL}$ or sTg $<1 \mathrm{ng} / \mathrm{mL}$ (in the absence of TgAb) concurrent with negative imaging at any time during the follow-up. Recurrence was defined as the detection of biochemical or structural evidence of disease following any disease-free period.

The final response to treatment was evaluated at the time of the final follow-up. Patients were classified as an excellent, incomplete biochemical, incomplete structural, or indeterminate response to treatment according to the previously reported response to therapy definitions and based on initial treatment $(15,16,17,18,29)$.

In patients who underwent total thyroidectomy and RAI treatment, the excellent response was defined as nonstimulated Tg $<0.2 \mathrm{ng} / \mathrm{mL}$ or $\mathrm{sTg}<1 \mathrm{ng} / \mathrm{mL}$ (in the absence of TgAb) and negative imaging. The incomplete biochemical response was defined as nonstimulated $\mathrm{Tg}$ $>1 \mathrm{ng} / \mathrm{mL}$ or $\mathrm{sTg}>10 \mathrm{ng} / \mathrm{mL}$ and negative imaging. The indeterminate response was defined as nonstimulated $\mathrm{Tg}$ $0.2-1 \mathrm{ng} / \mathrm{mL}$ or sTg $1-10 \mathrm{ng} / \mathrm{mL}$, or stable or declining antiTg levels and nonspecific imaging findings.
In patients who underwent thyroidectomy without RAI treatment, the excellent response was defined as nonstimulated $\mathrm{Tg}<0.2 \mathrm{ng} / \mathrm{mL}$ or $\mathrm{sTg}<2 \mathrm{ng} / \mathrm{mL}$ (in the absence of $\mathrm{TgAb}$ ) and negative imaging. An incomplete biochemical response was defined as nonstimulated Tg $>5$ $\mathrm{ng} / \mathrm{mL}$ or sTg $>10 \mathrm{ng} / \mathrm{mL}$, or rising Tg or anti-Tg levels over time and negative imaging. An indeterminate response was defined as nonstimulated Tg $0.2-5 \mathrm{ng} / \mathrm{mL}$ or sTg 2-10 ng/ $\mathrm{mL}$, or stable or declining anti-Tg levels and nonspecific imaging findings.

In patients who underwent lobectomy, an excellent response was defined as nonstimulated Tg $<30 \mathrm{ng} / \mathrm{mL}$ (in the absence of TgAb) and negative imaging. An incomplete biochemical response was defined as nonstimulated Tg $>30$ $n g / m L$ or rising Tg or anti-Tg levels over time and negative imaging. An indeterminate response was defined as stable or declining anti-Tg levels or nonspecific imaging findings.

An incomplete structural response was defined as evidence of structural and functional disease with any Tg or anti-Tg level, regardless of the extent of initial treatment.

\section{Evaluation of Outcomes}

Demographic data, clinicopathological features [history of irradiation, tumor size, subtypes of PTC, multifocality, lymphovascular invasion and extrathyroidal extension (ETE), and autoimmune thyroiditis], BRAF V600E mutation status, the extent of initial surgery, data related to RAI treatment, recurrence, and response to treatment were obtained. The clinicopathological features and clinical outcomes were assessed according to ATA risk levels and BRAF ${ }^{V 600 E}$ mutation. The correlations between the clinicopathological features, ATA initial risk level, $B R A F^{V 600 E}$ mutation, and recurrence and final response to treatment were analyzed.

The study was approved by the Ethics Committee of İstanbul University Faculty of Medicine (approval number: 478485, date: 21.09.2021).

\section{Statistical Analysis}

Continuous variables with normal distribution are reported as the mean \pm standard deviation (SD), non normal distribution as median (range), and categorical variables as numbers and percentages. The Student's t-test or MannWhitney $U$ test was used to compare the differences in continuous variables with normal or non-normal distribution, respectively. The chi-square test or Fisher's exact test was used in comparative analyses of categorical variables. A $p<0.05$ was considered to be statistically significant. Statistical analysis was performed using IBM Statistical Package for the Social Sciences Statistics for Windows, version 20.0 (IBM Corp., Armonk, NY, USA). 


\section{Results}

\section{Baseline Clinicopathological Characteristics and Treatment in Pediatric Patients with PTC}

The median age of the patients was 16 (5-18) years, with a female to male ratio of 55/15 (3.67:1). Eight (11\%) patients had a history of head and neck irradiation. The majority $(44 / 70,63 \%)$ of the patients presented with a solitary thyroid nodule. Fifteen ( $21 \%$ ) patients had palpable cervical lymph nodes at the time of initial diagnosis. Preoperative neck US revealed metastatic lymph nodes, in both the central and lateral neck in 15 (21\%), lateral neck only in five $(7 \%)$, and central neck only in three $(4 \%)$ patients. Cytologic examination of FNAB specimens of suspicious thyroid nodules revealed Bethesda 6 cytology in 37 (52\%), Bethesda 5 in 11 (16\%), Bethesda 4 in 11 (16\%), Bethesda 3 in seven $(10 \%)$, and benign in four $(6 \%)$ patients.

Total thyroidectomy was performed in $68(97 \%)$ patients, whereas two (3\%) patients underwent lobectomy. Lymph node dissection (LND), additional to total thyroidectomy, was done in $42(60 \%)$ patients. The types of LND were TCND with MRND in $21(50 \%)$, PCND in $18(43 \%)$, and TCND only in three $(7 \%)$ of 42 patients.

The median tumor size was 15 (3-50) mm. Histopathological examination revealed classical, follicular or aggressive variants of PTC in 28 (40\%), 29 (41\%), and 13 (19\%) patients, respectively. Forty-one patients $(59 \%)$ had multifocality, 17 (24\%) ETE, 31 (44\%) lymphovascular invasion, and 26 (37\%) autoimmune thyroiditis. Lymph node metastasis was found in 28 (40\%) patients. RAI treatment was performed in $52(74 \%)$ patients with a median $I^{131}$ dose of 125 (30-300 $\mathrm{mCi}$ ). Distant metastasis to the lungs was detected in five (7\%) patients on WBS after postoperative RAI treatment. The median follow-up was 6 (2-21.8) years.

Twenty locoregional recurrences developed in 12 (17\%) patients during the follow-up. No recurrence at distant sites was observed. When the risk of locoregional recurrence according to clinicopathological factors was analyzed, classical variant of PTC $(p=0.001)$, ETE $(p<0.001)$, lymphovascular invasion $(p<0.001)$, and lymph node metastasis $(p=0.001)$ were significant risk factors (Table 1). The total dose of $I^{131}$ administered for RAI treatment in patients who had recurrence was significantly higher than the patients with no recurrence $(p<0.001)$ (Table 1). There was no disease-related mortality.

\section{ATA Initial Pediatric Risk Stratification}

According to the ATA initial pediatric risk stratification, 44 $(63 \%), 14(20 \%)$, and $12(17 \%)$ patients were classified as low-, intermediate-, and high-risk, respectively. The comparison of clinicopathological features and clinical outcomes in ATA low-risk patients vs intermediate- and highrisk patients are summarized in Table 2. An ATA low-risk state was significantly associated with smaller tumor size, lower rates of aggressive variant PTC, multifocality, ETE, lymphovascular invasion, lymph node metastasis, LND, RAI treatment and locoregional recurrence, and a higher rate of follicular variant PTC (FVPTC) compared to paptients classified as ATA intermediate- and high-risk (Table 2). None of the ATA low-risk patients had distant metastasis, whereas lung metastasis was observed in $19 \%$ of ATA intermediateand high-risk patients $(p=0.003)$.

All intermediate- and high-risk patients underwent total thyroidectomy, whereas lobectomy was performed in two (4\%) of 44 low-risk patients. The median total dose of administered $I^{131}$ was significantly higher in intermediateand high-risk vs low-risk patients $(150 \mathrm{mCi}$ vs $67.5 \mathrm{mCi}$, respectively; $p<0.001$ ) (Table 2 ).

\section{Final Response to Treatment}

Response to treatment according to DRS at the end of followup revealed excellent response in 61 (87\%), incomplete biochemical response in two $(3 \%)$, indeterminate response in one (1\%), and incomplete structural response (persistent disease) in six (9\%) patients. Five of the six patients with persistent disease were ATA high-risk patients and the rate of persistent disease in the high-risk group was $42 \%(5 / 12)$. The rate of excellent response was $100 \%, 93 \%$, and $33 \%$ in ATA low-, intermediate-, and high-risk levels, respectively. The rate of excellent response was significantly lower in ATA intermediate- and high-risk patients when compared to ATA low- risk patients $(65.4 \%$ vs $100 \%$, p < 0.001) (Table 2$)$. When we compared the clinicopathological features in patients with and without excellent response, older age, FVPTC, unifocality, absence of lymphovascular invasion, initial lymph node metastasis or distant metastasis, and ATA lowrisk significantly predicted excellent response to treatment (Table 3). The total dose of I ${ }^{131}$ used for RAI treatment was lower in patients with excellent response compared to those without excellent response, but the difference did not achieve statistical significance $(p=0.055)$ (Table 3$)$. The patients who developed locoregional recurrences during the follow-up showed a significantly lower rate of excellent response to treatment at final follow-up compared to those without recurrences (50\% vs $95 \%, \mathrm{p}<0.001$ ) (Table 1 ).

\section{BRAF ${ }^{V 600 E}$ Mutation Status}

BRAF ${ }^{V 600 E}$ mutation was positive in 18 (33\%) of 55 patients with available data. The median age of these 55 patients 
was 16 (5-18) years. The median (range) tumor size was 14 (4-50) $\mathrm{mm}$. The correlation between the BRAFV600E mutation status and clinicopathological features, ATA initial risk stratification, the extent of surgery and clinical outcomes are summarized in Table 4. Age, gender, history of irradiation and tumor size showed no significant difference between the patients with or without the mutation. Classical variant PTC was significantly associated with the presence of the $B R A F^{V 600 E}$ mutation $(\mathrm{p}=0.01)$, whereas the rate of FVPTC was significantly higher in $B R A F^{V 600 E}(-)$ patients compared to $B_{R A F^{V 600 E}}(+)$ patients $(p=0.01)$. The rate of the aggressive variant of PTC showed no significant difference between the $B R A F^{V 600 E}(+)$ and $(-)$ patients. Although the rate of multifocality was higher in $\operatorname{BRAF}^{V 600 E}(+)$ patients compared to $B R A F^{V 600 E}$ (-) patients, the difference did not achieve statistical significance ( $83 \%$ vs $57 \%$, respectively; $\mathrm{p}=0.052$ ). There was no correlation between $B R A F^{V 600 E}$ mutation and lymphovascular invasion, ETE, autoimmune thyroiditis, the extent of thyroidectomy, LND, lymph node metastasis, distant metastasis, and ATA initial risk levels (Table 4). The recurrence rate was significantly higher in $B A^{V} F^{V O O E}(+)$ patients compared to BRAF ${ }^{V 600 E}$ (-) patients
(33\% vs $3 \%, p=0.001$ ). A total of 14 recurrences were observed in six of $18 B R A F^{V 600 E}(+)$ patients, whereas there was only one recurrence in one of $37 B R A F^{V 600 E}(-)$ patients $(p<0.001)$. The total dose of $\mathrm{I}^{131}$ used for RAI treatment was significantly higher in $B R A F^{V 600 E}(+)$ compared to BRAF ${ }^{V 600 E}$ $(-)$ patients $(\mathrm{p}<0.001)$. The rate of excellent response in $B_{R A F^{V O O O E}}(+)$ and $(-)$ patients were $83 \%$ and $92 \%$, respectively, and showed no significant difference $(p=0.3)$. The biochemical incomplete, indeterminate and structural incomplete response rates showed no significant difference between the BRAF V600E $(+)$ and (-) patients (Table 4).

\section{Discussion}

In our study, we found that all ATA low-risk and $93 \%$ of intermediate-risk patients had an excellent response to treatment at final follow-up. The presence of BRAF V600E mutation was highly predictive for locoregional recurrence but had no significant effect on the final rate of excellent response to treatment.

Thyroid cancer is rare in children, and there is a limited number of studies with a large number of patients. The

Table 1. The clinicopathological features and clinical outcomes in patients with and without locoregional recurrences

\begin{tabular}{|c|c|c|c|}
\hline & Recurrence $(+)(n=12)$ & Recurrence $(-)(n=58)$ & $\mathrm{p}$ \\
\hline Median (range) age, years & $14.5(8-18)$ & $16(6-18)$ & 0.3 \\
\hline \multicolumn{4}{|l|}{ Gender, n (\%) } \\
\hline Female & $9(75)$ & $46(79)$ & \multirow[t]{2}{*}{0.7} \\
\hline Male & $3(25)$ & $12(21)$ & \\
\hline History of irradiation, n (\%) & $0(0)$ & $8(14)$ & 0.1 \\
\hline Tumor size, mm. & $20.5(9-42)$ & $14(3-50)$ & 0.1 \\
\hline \multicolumn{4}{|l|}{ Histologic type, n (\%) } \\
\hline Classical & $10(83)$ & $18(31)$ & 0.001 \\
\hline Folicular & $0(0)$ & $29(50)$ & 0.001 \\
\hline Aggresive & $2(17)$ & $11(19)$ & 0.8 \\
\hline \multicolumn{4}{|l|}{ Pathological features, n (\%) } \\
\hline Multifocality & $10(83)$ & $31(53)$ & 0.056 \\
\hline Extrathyroidial extension & $9(75)$ & $8(14)$ & 0.0001 \\
\hline Lymphovascular invasion & $11(92)$ & $20(34)$ & $<0.001$ \\
\hline Autoimmune thyroiditis & $4(33)$ & $22(38)$ & 0.6 \\
\hline Total thyroidectomy, n (\%) & $12(100)$ & $56(97)$ & 0.5 \\
\hline Total LND, n (\%) & $10(83)$ & $32(55)$ & 0.06 \\
\hline TCND + MRND, n (\%) & $10(83)$ & $11(19)$ & $<0.001$ \\
\hline Lymph node metastasis, n (\%) & $10(83)$ & $18(31)$ & 0.001 \\
\hline Distant metastasis, n (\%) & $2(17)$ & $3(5)$ & 0.1 \\
\hline ATA low risk, n (\%) & $1(8)$ & $43(74)$ & $<0.001$ \\
\hline RAI treatment, n (\%) & $12(100)$ & $40(69)$ & 0.02 \\
\hline Median (range) total I $\mathrm{I}^{131}$ dose (mCi) & $150(60-300)$ & $100(30-150)$ & $<0.001$ \\
\hline Excellent response, n (\%) & $6(50)$ & $55(95)$ & $<0.001$ \\
\hline
\end{tabular}


initial presentation, clinical course, and mortality of PTC in children shows major differences compared to adult patients. The rate of multifocal disease ranged between $28 \%$ and $57 \%$, ETE between $36 \%$ and $59 \%$, central and/or lateral neck metastasis between $60 \%$ and $70 \%$, and initial distant metastasis between $4.7 \%$ and $14.6 \%$ in pediatric DTC $(14,24,30,31)$. Classical variants constitute the majority of PTC in pediatric and adolescent patients $(13,14,24,30,31)$. Although the initial presentation of childhood DTC is more severe compared to adults, the long-term outcome is favorable, with very low mortality rates $(3,7)$. We found a high rate $(41 \%)$ of FVPTC in our cohort. In a former study from our instution, the rate of FVPTC was reported as $37.2 \%$ in adult PTC patients (32). The reported rates of FVPTC in pediatric PTC patients ranged between $10.4 \%$ and $36.8 \%$, and was $22.7 \%$ in a large database study, which included 1,956 pediatric patients $(24,30,31,33,34)$. The relatively high rate of FVPTC in our pediatric patients might be an incidental finding in a particulary small cohort or might be a reflection of regional and environmental differences in PTC features.

The local or distant recurrence rates are reported to range between 15.9-34\% in pediatric and adolescent DTC patients $(13,14,30,35)$. Recurrence was significantly associated with multifocality, large tumors, palpable cervical lymph nodes, lymph node metastasis, ETE or distant metastasis at diagnosis in pediatric and adolescent PTC $(30,35)$. In the study by Welch Dinauer et al (35), the authors showed that focality was the best predictor of recurrence by multivariate analysis. In our study, the increased rate of locoregional recurrence was significantly associated with classical variant PTC, ETE, lymphovascular invasion, lymph node metastasis, and the presence of BRAF ${ }^{V 600 E}$ mutation. However, age, gender, autoimmune thyroiditis,

Table 2. The comparison of the clinicopathological features, extent of surgery and clinical outcomes in ATA pediatric low-and intermediate/high-risk patients

\begin{tabular}{|c|c|c|c|}
\hline & ATA low risk $(n=44)$ & ATA intermediate and high risk $(n=26)$ & $\mathrm{p}$ \\
\hline Median (range) age, years & $16(6-18)$ & $15.5(5-18)$ & 0.9 \\
\hline \multicolumn{4}{|l|}{ Gender, n (\%) } \\
\hline $\begin{array}{l}\text { Female } \\
\text { Male }\end{array}$ & $\begin{array}{l}35(79.5) \\
9(20.5)\end{array}$ & $\begin{array}{l}20(77) \\
6(23)\end{array}$ & 0.8 \\
\hline History of irradiation, n (\%) & $8(18)$ & $0(0)$ & 0.02 \\
\hline Median (range) tumor size, $\mathrm{mm}$ & $10.5(3-50)$ & $21(9-45)$ & 0.001 \\
\hline \multicolumn{4}{|l|}{ Histologic type, n (\%) } \\
\hline Classical & $14(32)$ & $14(53.8)$ & 0.07 \\
\hline Folicular & $27(61)$ & $2(7.7)$ & $<0.001$ \\
\hline Aggresive & $3(7)$ & $10(38.5)$ & 0.001 \\
\hline \multicolumn{4}{|l|}{ Pathological features, n (\%) } \\
\hline Multifocality & $21(48)$ & $20(80)$ & 0.017 \\
\hline Extrathyroidial extension & $0(0)$ & $17(65.4)$ & $<0.001$ \\
\hline Lymphovascular invasion & $7(16)$ & $24(92.3)$ & $<0.001$ \\
\hline Autoimmune thyroiditis & $18(41)$ & $8(31)$ & 0.4 \\
\hline Total thyroidectomy, n (\%) & $42(96)$ & $26(100)$ & 0.2 \\
\hline Total LND, n (\%) & $17(39)$ & $25(96)$ & $<0.001$ \\
\hline TCND + MRND, n (\%) & $1(2.3)$ & $20(80)$ & $<0.001$ \\
\hline Lymph node metastasis, n (\%) & $4(9)$ & $24(92.3)$ & $<0.001$ \\
\hline Distant metastasis, n (\%) & $0(0)$ & $5(19)$ & 0.003 \\
\hline RAI treatment, $\mathrm{n}(\%)$ & $26(59)$ & $26(100)$ & $<0.001$ \\
\hline Median (range) total I $\mathrm{I}^{131}$ dose (mCi) & $67.5(30-300)$ & $150(50-300)$ & $<0.001$ \\
\hline Recurrence, n (\%) & $1(2.2)$ & $11(42.3)$ & $<0.001$ \\
\hline Number of recurrences, $\mathrm{n}(\%)$ & $1(2.3)$ & $19(73)$ & 0.003 \\
\hline \multicolumn{4}{|l|}{ Final DRS, n (\%) } \\
\hline Excellent response & $44(100)$ & $17(65.4)$ & $<0.001$ \\
\hline Biochemical incomplete/indeterminate & $0(0)$ & $3(11.5)$ & 0.047 \\
\hline Structural incomplete & $0(0)$ & $6(23.1)$ & 0.002 \\
\hline
\end{tabular}

LND: lymph node dissection, TCND: therapeutic central neck dissection, MRND: modified radical neck dissection, RAI: radioactive iodine, ATA: American Thyroid Association, DRS: dynamic risk stratification 
tumor size, and distant metastasis were not significantly associated with local recurrence in our cohort. Although the rate of multifocality was approximately 1.5 -fold higher in patients who developed locoregional recurrence compared to patients with no recurrence, the difference was not statistically significant.

Recently, the ATA recommended that pediatric PTC patients should be initially stratified into ATA pediatric low-, intermediate-, or high-risk levels, based on clinical presentation, tumor size, and evidence of regional invasion and metastasis to identify the patients at risk of persistent or recurrent cervical disease (1). The ATA's initial pediatric risk stratification system has been validated by several studies, and the findings of our study were similar to the results of these other studies $(11,12,13,14,20)$. We observed significantly higher rates of locoregional recurrence and persistent disease in ATA intermediate- and high-risk patients compared to ATA low-risk patients.

The ATA 2015 Modified Risk Stratification System for adult DTC patients does not suggest a routine analysis of $B R A F^{V 600 E}$ status for initial risk stratification (8). However, BRAFV600E mutation has been included in the continuous risk scale for the assessment of the risk of structural disease in adults to help clinicians to perform proper risk stratification in cases where mutation information is available (8). The rate of mutation positivity exhibited great difference according to the age of the patient in a pediatric population and was very low in younger children $(26,27)$. In the study by Nikita et al (27), $89 \%$ of BRAF ${ }^{V 600 E}$ mutations were detected in patients older than 15 years and $11 \%$ in those younger than 15 years old. In our study, we observed that the median age of $B R A F^{V 600 E}(+)$ patients was older compared to BRAF ${ }^{V 600 E}(-)$ patients, but the difference was not significant. The studies that evaluated the correlation between $B R A F^{V 600 E}$ mutation and histopathological features, the extent of disease, and prognosis in pediatric PTC patients showed that BRAF ${ }^{\text {V600E }}$ mutation was not significantly associated with adverse histopathological features, lymph node metastasis, or distant metastasis and did not predict an aggressive clinical course, as it does in adult PTC $(24,25,31,33)$. BRAFVG00E mutation was found to be very frequent in classical variant PTC compared to non-classical variants in several studies $(25,31,33)$. Similarly, we observed that the rate of classical variant PTC was approximately 2 -fold higher in $B R A F^{V 600 E}$ $(+)$ patients compared to BRAF ${ }^{V 600 E}(-)$ patients, in our

\begin{tabular}{|c|c|c|c|}
\hline & Excellent response $(+)(n=61)$ & Excellent response $(-)(n=9)$ & $\mathrm{p}$ \\
\hline Median (range) age, years & $16(6-18)$ & $13(5-18)$ & 0.045 \\
\hline \multicolumn{4}{|l|}{ Gender, n (\%) } \\
\hline $\begin{array}{l}\text { Female } \\
\text { Male }\end{array}$ & $\begin{array}{l}49(80) \\
12(20)\end{array}$ & $\begin{array}{l}6(67) \\
3(33)\end{array}$ & 0.3 \\
\hline History of irradiation, n (\%) & 8 & 0 & 0.2 \\
\hline Median (range) tumor size, $\mathrm{mm}$ & $14(3-50)$ & $20(9-45)$ & 0.2 \\
\hline \multicolumn{4}{|l|}{ Histologic type, $\mathrm{n}(\%)$} \\
\hline Classical & $22(36)$ & $6(67)$ & 0.08 \\
\hline Folicular & $29(48)$ & $0(0)$ & 0.007 \\
\hline Aggresive & $10(16)$ & $3(33)$ & 0.2 \\
\hline \multicolumn{4}{|l|}{ Pathological features, $\mathrm{n}(\%)$} \\
\hline Multifocality & $32(52)$ & $9(100)$ & 0.007 \\
\hline Extrathyroidial extension & $10(48)$ & $7(78)$ & $<0.001$ \\
\hline Lymphovascular invasion & $23(38)$ & $8(89)$ & 0.004 \\
\hline Autoimmune thyroiditis & $23(239$ & $3(339$ & 0.8 \\
\hline Total thyroidectomy, n (\%) & 59 (98) & $9(100)$ & 0.5 \\
\hline Total LND, n (\%) & $33(54)$ & $9(100)$ & 0.008 \\
\hline TCND + MRND, n (\%) & $12(16)$ & $9(100)$ & $<0.001$ \\
\hline Lymph node metastasis, n (\%) & $19(319$ & $9(100)$ & $<0.001$ \\
\hline Distant metastasis, n (\%) & $0(0)$ & $5(56)$ & $<0.001$ \\
\hline Recurrence, n (\%) & $6(9.8)$ & $6(67)$ & $<0.001$ \\
\hline ATA low risk, n (\%) & $44(72)$ & $0(0)$ & $<0.001$ \\
\hline RAI treatment, $\mathrm{n}(\%)$ & $43(70)$ & $9(100)$ & 0.06 \\
\hline Median (range) total $\mathrm{I}^{131}$ dose $(\mathrm{mCi})$ & $100(30-300)$ & $150(50-300)$ & 0.055 \\
\hline
\end{tabular}

LND: lymph node dissection, TCND: therapeutic central neck dissection, MRND: modified radical neck dissection, RAI: radioactive iodine 
study. Geng et al (24) showed that the BRAF ${ }^{V 600 E}$ mutation was significantly associated with both a low AJCC and low AMES tumor stage. The authors reported the rates of BRAF ${ }^{V 600 E}$ mutation as $63.6 \%, 40 \%$, and $22.2 \%$ in ATA low, intermediate-, and high-risk patients, respectively, with no statistical difference according to the risk level (24). In our study, we also found no significant correlation between the BRAF ${ }^{V O O O E}$ mutation and ATA pediatric initial risk levels. Nor did we observe any significant correlation between the $B R A F^{V 600 E}$ mutation and adverse histopathological features and initial presentation of PTC. Although, $B R A F^{V 600 E}$ mutation was not associated with unfavorable clinicopathological risk factors initially, we observed that it was a significant predictive factor for recurrence in our patients. In our study, the rates of locoregional recurrence in $B R A F^{V 600 E}(+)$ vs (-) patients were $33 \%$ vs $3 \%$, respectively.
The DRS has been proposed for re-staging patients according to response to treatment by re-evaluating the clinical, biochemical, imaging, and cytopathologic findings at any time during follow-up $(8,15,16,17,18)$. The DRS has been validated in patients treated with total thyroidectomy and RAI treatment, and a modified DRS system could also be applied to DTC patients who underwent lobectomy or total thyroidectomy without RAI ablation $(15,18,29)$.

Sohn et al (20) showed that the prevalence of structural persistent disease increased as ATA initial risk classification increased in pediatric DTC. Other studies have reported that low-risk patients had the highest probability of an excellent response to initial treatment while high-risk patients had the highest probability of incomplete structural response and the lowest probability of an excellent response $(13,14)$. Our findings were compatible with these studies. We found the

Table 4. The comparison of clinicopathological features, extent of surgery, ATA initial risk stratification, and response to treatment according to $B R A F^{V 600 E}$ mutation status

\begin{tabular}{|c|c|c|c|}
\hline & $B R A F^{V 600 E}(+)(\mathrm{n}=18)$ & $B R A F^{V 600 E}(-)(\mathrm{n}=37)$ & $\mathrm{p}$ \\
\hline Median (range) age, years & $16(14-18)$ & $15(5-18)$ & 0.064 \\
\hline \multicolumn{4}{|l|}{ Gender, n (\%) } \\
\hline $\begin{array}{l}\text { Female } \\
\text { Male }\end{array}$ & $\begin{array}{l}15(83) \\
3(17)\end{array}$ & $\begin{array}{l}29(78.4) \\
8(21.6)\end{array}$ & 0.6 \\
\hline History of irradiation, n (\%) & $0(0)$ & $6(16)$ & 0.07 \\
\hline Median (range) tumor size, $\mathrm{mm}$ & $15(6-50)$ & $14(4-50)$ & 0.4 \\
\hline \multicolumn{4}{|l|}{ Histologic type, n (\%) } \\
\hline Classical & $11(61)$ & $10(27)$ & 0.01 \\
\hline Folicular & $4(22)$ & $21(57)$ & 0.01 \\
\hline Aggresive & $3(7)$ & $6(16)$ & 0.9 \\
\hline \multicolumn{4}{|l|}{ Pathological features, n (\%) } \\
\hline Multifocality & $15(83)$ & $21(57)$ & 0.052 \\
\hline Extrathyroidial extension & $6(33)$ & $8(22)$ & 0.3 \\
\hline Lymphovascular invasion & $10(55)$ & $12(32)$ & 0.1 \\
\hline Autoimmune thyroiditis & $8(44)$ & $14(38)$ & 0.6 \\
\hline Total thyroidectomy, n (\%) & $18(100)$ & $36(97)$ & 0.48 \\
\hline Total LND, n (\%) & $12(67)$ & $23(62)$ & 0.75 \\
\hline TCND + MRND, n (\%) & $6(33)$ & $10(27)$ & 0.6 \\
\hline Lymph node metastasis, n (\%) & $9(50)$ & $13(35)$ & 0.2 \\
\hline Distant metastasis, n (\%) & $0(0)$ & $3(8)$ & 0.2 \\
\hline ATA low risk, n (\%) & $10(55)$ & $27(73)$ & 0.1 \\
\hline $\mathrm{RAI}^{\dagger}$ treatment, $\mathrm{n}(\%)$ & $16(89)$ & $25(68)$ & 0.09 \\
\hline Median (range) total dose of $\mathrm{I}^{131}(\mathrm{mCi})$ & $150(100-300)$ & $75(30-250)$ & 0.001 \\
\hline Patients with recurrence, $\mathrm{n}(\%)$ & $6(33)$ & $1(3)$ & 0.001 \\
\hline Number of recurrences, $\mathrm{n}(\%)$ & $14(78)$ & $1(3)$ & $<0.001$ \\
\hline \multicolumn{4}{|l|}{ DRS Response to treatment, $\mathrm{n}(\%)$} \\
\hline Excellent & $15(83)$ & $34(92)$ & 0.3 \\
\hline Biochemical incomplete/indeterminate & $2(11)$ & $1(2.7)$ & 0.2 \\
\hline Structural incompelete & $1(6)$ & $2(5.3)$ & 0.9 \\
\hline
\end{tabular}

LND: lymph node dissection, TCND: therapeutic central neck dissection, MRND: modified radical neck dissection, RAI': radioactive iodine, ATA: American Thyroid Association, DRS: dynamic risk stratification performed at the end of the follow up 
rate of excellent response to be $100 \%, 93 \%$ and $33 \%$ in ATA low-, intermediate- and high-risk patients, respectively. In our study, excellent response to treatment was significantly associated with ATA low-risk, older age, FVPTC, unifocal tumors with no invasion and metastasis, and no recurrences during the follow-up.

The impact of $B R A F^{V 600 E}$ mutation status on response to treatment evaluated by DRS has not been previously investigated in pediatric PTC patients. When we analyzed the response to treatment in $B R A F^{V 600 E}(+)$ and (-) patients, no significant difference was found between the two groups in respect of excellent response to treatment at final follow-up. Although the rate of biochemical incomplete/indeterminate response was higher in $B R A F^{V 600 E}(+)$ patients compared to $B R A F^{V 600 E}(-)$ patients, the difference was not statistically significant ( $11 \%$ vs $2.7 \%, p=0.2$ ). Our findings suggest that $B R A F^{V 600 E}$ mutation might be associated with a higher rate of locoregional recurrence but probably do not increase the long-term risk of incomplete structural response to treatment.

Some studies have reported that younger age was found to be associated with initial high risk and recurrent/persistent disease in pediatric PTC, while others found no correlation with age and prognosis $(12,36,37,38,39)$. We observed that older age was significantly associated with excellent response to treatment. Gender was not associated with either the initial risk stratification or response to treatment in our study and this finding was compatible with the studies in the literature $(36,37,38,39)$.

\section{Study Limitations}

This study has some limitations. This is a retrospective study with a relatively small sample size. Pediatric DTC is a rare disease, and studies reporting outcomes of more than 100 children are few. There is the possibility of selection bias as all of the patients in this study were treated in a single tertiary referral center. BRAF ${ }^{V 600 E}$ analysis was performed in $78.5 \%$ of the cohort. The small sample size might be insufficient to determine the correlation between BRAF ${ }^{V 600 E}$ mutation and recurrence or response to treatment.

\section{Conclusion}

We showed that ATA initial pediatric risk stratification effectively predicted the risk of recurrent and persistent disease and final response to treatment in PTC patients $\leq 18$ years old. All ATA low-risk and $93 \%$ of intermediaterisk patients had an excellent response to treatment at final follow-up. The presence of BRAF ${ }^{V 600 E}$ mutation was highly predictive for locoregional recurrence but had no significant effect on the final rate of excellent response to treatment. During initial risk evaluation of pediatric PTC patients, investigation of BRAF ${ }^{V 600 E}$ mutation status in addition to ATA initial stratification might provide a better estimate of the probability of recurrence in those patients in whom $B R A F^{V 600 E}$ mutation status can be determined. Further studies with a large number of patients are needed to determine the role of the BRAF ${ }^{V 600 E}$ mutation on recurrence and response to treatment in pediatric PTC.

\section{Ethics}

Ethics Committee Approval: The study was approved by the Ethics Committee of İstanbul University Faculty of Medicine (approval number: 478485, date: 21.09.2021).

Informed Consent: Retrospective study.

Peer-review: Externally peer-reviewed.

\section{Authorship Contributions}

Surgical and Medical Practices: Yasemin Giles Şenyürek, Yalın İşcan, İsmail Cem Sormaz, Şükran Poyrazoğlu, Fatih Tunca, Concept: Yasemin Giles Şenyürek, Yalın İşcan, Fatih Tunca, Design: Yasemin Giles Şenyürek, Fatih Tunca, Data Collection or Processing: Yasemin Giles Şenyürek, Yalın İşcan, İsmail Cem Sormaz, Analysis or Interpretation: Yasemin Giles Şenyürek, Fatih Tunca, Literature Search: Yasemin Giles Şenyürek, Şükran Poyrazoğlu, Writing: Yasemin Giles Şenyürek, Fatih Tunca.

Financial Disclosure: The authors declared that this study received no financial support.

\section{References}

1. Francis GL, Waguespack SG, Bauer AJ, Angelos P, Benvenga S, Cerutti JM, Dinauer CA, Hamilton J, Hay ID, Luster M, Parisi MT, Rachmiel M, Thompson GB, Yamashita S; American Thyroid Association Guidelines Task Force. Management Guidelines for Children with Thyroid Nodules and Differentiated Thyroid Cancer. Thyroid 2015;25:716-759.

2. Drozd V, Saenko V, Branovan DI, Brown K, Yamashita S, Reiners C. A search for causes of rising incidence of differentiated thyroid cancer in children and adolescents after Chernobyl and Fukushima: Comparison of the clinical features and their relevance for treatment and prognosis. Int J Environ Res Public Health 2021;18:3444.

3. Vaccarella S, Lortet-Tieulent J, Colombet M, Davies L, Stiller CA, Schüz J, Togawa K, Bray F, Franceschi S, Dal Maso L, Steliarova-Foucher E; IICC3 contributors. Global patterns and trends in incidence and mortality of thyroid cancer in children and adolescents: a population-based study. Lancet Diabetes Endocrinol 2021;9:144-152. Epub 2021 Jan 19

4. Bleyer WA. Cancer in older adolescents and young adults: epidemiology, diagnosis, treatment, survival, and importance of clinical trials. Med Pediatr Oncol 2002;38:1-10.

5. Bernier MO, Withrow DR, Berrington de Gonzalez A, Lam CJK, Linet MS, Kitahara CM, Shiels MS. Trends in pediatric thyroid cancer incidence in the United States, 1998-2013. Cancer 2019;125:2497-2505. Epub 2019 Apr 23 
6. Rivkees SA, Mazzaferri EL, Verburg FA, Reiners C, Luster M, Breuer CK, Dinauer CA, Udelsman R. The treatment of differentiated thyroid cancer in children: Emphasis on surgical approach and radioactive iodine therapy. Endocr Rev 2011;32:798-826. Epub 2011 Aug 31

7. Qian ZJ, Jin MC, Meister KD, Megwalu UC. Pediatric thyroid cancer incidence and mortality trends in the United States, 1973-2013. JAMA Otolaryngol Head Neck Surg 2019;145:617-623.

8. Haugen BR, Alexander EK, Bible KC, Doherty GM, Mandel SJ, Nikiforov YE, Pacini F, Randolph GW, Sawka AM, Schlumberger M, Schuff KG, Sherman SI, Sosa JA, Steward DL, Tuttle RM, Wartofsky L. 2015 American Thyroid Association Management Guidelines for Adult Patients with Thyroid Nodules and Differentiated Thyroid Cancer: The American Thyroid Association Guidelines Task Force on Thyroid Nodules and Differentiated Thyroid Cancer. Thyroid 2016;26:1-133.

9. Amin M, Edge S, Greene F. AJCC cancer staging manual. 8th. New York, Springer, 2017.

10. Karapanou O, Tzanela M, Rondogianni P, Dacou-Voutetakis C, Chiotis D, Vlassopoulou B, Vassiliadi D, Kanaka-Gantenbein C, Tsagarakis S. Long-term outcome of differentiated thyroid cancer in children and young adults: risk stratification by ATA criteria and assessment of preablation stimulated thyroglobulin as predictors of disease persistence. Endocrine 2020;70:566-574. Epub 2020 Jun 13

11. Pires BP, Alves PA Jr, Bordallo MA, Bulzico DA, Lopes FP, Farias T, Dias F, Lima RA, Santos Gisler IC, Coeli CM, Carvalhaes de Oliveira RV, Corbo R, Vaisman M, Vaisman F. Prognostic factors for early and longterm remission in pediatric differentiated thyroid carcinoma: The role of sex, age, clinical presentation, and the newly proposed American Thyroid Association Risk Stratification System. Thyroid 2016;26:14801487. Epub 2016 Sep 22

12. Lazar L, Lebenthal Y, Segal K, Steinmetz A, Strenov Y, Cohen M, Yaniv I, Yackobovitch-Gavan M, Phillip M. Pediatric thyroid cancer: postoperative classifications and response to initial therapy as prognostic factors. J Clin Endocrinol Metab 2016;101:1970-1979. Epub 2016 Mar 1

13. Sung TY, Jeon MJ, Lee YH, Lee YM, Kwon H, Yoon JH, Chung KW, Kim WG, Song DE, Hong SJ. Initial and dynamic risk stratification of pediatric patients with differentiated thyroid cancer. J Clin Endocrinol Metab 2017;102:793-800.

14. Kim K, Kim WW, Choi JB, Kim MJ, Lee CR, Lee J, Kang SW, Nam $\mathrm{KH}$, Chung WY, Jeong JJ. Usefulness of dynamic risk stratification in pediatric patients with differentiated thyroid carcinoma. Ann Surg Treat Res 2018;95:222-229. Epub 2018 Sep 28

15. Tuttle RM, Tala H, Shah J, Leboeuf R, Ghossein R, Gonen M, Brokhin M, Omry G, Fagin JA, Shaha A. Estimating risk of recurrence in differentiated thyroid cancer after total thyroidectomy and radioactive iodine remnant ablation: using response to therapy variables tomodify the initial risk estimates predicted by the new American Thyroid Association staging system. Thyroid 2010;20:1341-1349. Epub 2010 Oct 29

16. Vaisman F, Momesso D, Bulzico DA, Pessoa CH, Dias F, Corbo R, Vaisman M, Tuttle RM. Spontaneous remission in thyroid cancer patients after biochemical incomplete response to initial therapy. Clin Endocrinol (Oxf) 2012;77:132-138.

17. Castagna MG, Maino F, Cipri C, Belardini V, Theodoropoulou A, Cevenini G, Pacini F. Delayed risk stratification, to include the response to initial treatment (surgery and radioiodine ablation), has better outcome predictivity in differentiated thyroid cancer patients. Eur J Endocrinol 2011;165:441-446. Epub 2011 Jul 12

18. Pitoia F, Bueno F, Urciuoli C, Abelleira E, Cross G, Tuttle RM. Outcomes of patients with differentiated thyroid cancer risk-stratified according to the American Thyroid Association and Latin American Thyroid Society risk of recurrence classification systems. Thyroid 2013;23:1401-1407. Epub 2013 Jul 25
19. Zanella AB, Scheffel RS, Nava CF, Golbert L, Laurini de Souza Meyer E, Punales M, Gonçalves I, Dora JM, Maia AL. Dynamic risk stratification in the follow-up of children and adolescents with differentiated thyroid cancer. Thyroid 2018;28:1285-1292.

20. Sohn SY, Kim YN, Kim HI, Kim TH, Kim SW, Chung JH. Validation of dynamic risk stratification in pediatric differentiated thyroid cancer. Endocrine 2017;58:167-175. Epub 2017 Aug 18

21. Tufano RP, Teixeira GV, Bishop J, Carson KA, Xing M. BRAF mutation in papillary thyroid cancer and its value in tailoring initial treatment: A systematic review and meta-analysis. Medicine (Baltimore) 2012;91:274-286.

22. Wang Z, Chen JQ, Liu JL, Qin XG. Clinical impact of BRAF mutation on the diagnosis and prognosis of papillary thyroid carcinoma: A systematic review and meta-analysis. Eur J Clin Invest 2016;46:146157. Epub 2016 Jan 18

23. Galuppini F, Vianello F, Censi S, Barollo S, Bertazza L, Carducci S, Colato C, Manso J, Rugge M, Iacobone M, Watutantrige Fernando S, Pennelli G, Mian C. Differentiated thyroid carcinoma in pediatric age: Genetic and clinical scenario. Front Endocrinol (Lausanne) 2019;10:552.

24. Geng J, Wang H, Liu Y, Tai J, Jin Y, Zhang J, He L, Fu L, Qin H, Song Y, Su J, Zhang A, Wen X, Guo Y, Ni X. Correlation between BRAF V600E mutation and clinicopathological features in pediatric papillary thyroid carcinoma. Sci China Life Sci 2017;60:729-738. Epub 2017 Jun 15

25. Hardee S, Prasad ML, Hui P, Dinauer CA, Morotti RA. Pathologic characteristics, natural history, and prognostic implications of BRAFV600E mutation in pediatric papillary thyroid carcinoma. Pediatr Dev Pathol 2017;20:206-212. Epub 2017 Feb 8

26. Poyrazoğlu Ş, Bundak R, Baş F, Yeğen G, Şanlı Y, Darendeliler F. Clinicopathological characteristics of papillary thyroid cancer in children with emphasis on pubertal status and association with BRAF V600E mutation. J Clin Res Pediatr Endocrinol 2017;9:185-193. Epub 2017 Jan 12

27. Nikita ME, Jiang W, Cheng SM, Hantash FM, McPhaul MJ, Newbury RO, Phillips SA, Reitz RE, Waldman FM, Newfield RS. Mutational analysis in pediatric thyroid cancer and correlations with age, ethnicity, and clinical presentation. Thyroid 2016;26:227-234. Epub 2016 Jan 7

28. Vaughn CP, Zobell SD, Furtado LV, Baker CL, Samowitz WS. Frequency of KRAS, BRAF, and NRAS mutations in colorectal cancer. Genes Chromosomes Cancer 2011;50:307-312. Epub 2011 Feb 8

29. Momesso DP, Tuttle RM. Update on differentiated thyroid cancer staging. Endocrinol Metab Clin North Am 2014;43:401-421.

30. Grigsby PW, Gal-or A, Michalski JM, Doherty GM. Childhood and adolescent thyroid carcinoma. Cancer 2002;95:724-729.

31. Henke LE, Perkins SM, Pfeifer JD, Ma C, Chen Y, DeWees T, Grigsby PW. BRAF V600E mutational status in pediatric thyroid cancer. Pediatr Blood Cancer 2014;61:1168-1172. Epub 2014 Mar 27

32. Tunca F, Sormaz IC, Iscan Y, Senyurek YG, Terzioglu T. Comparison of histopathological features and prognosis of classical and follicular variant papillary thyroid carcinoma. J Endocrinol Invest 2015;38:13271334. Epub 2015 Aug 18

33. Givens DJ, Buchmann LO, Agarwal AM, Grimmer JF, Hunt JP. BRAF V600E does not predict aggressive features of pediatric papillary thyroid carcinoma. Laryngoscope 2014;124:389-393. Epub 2014 Apr 29

34. Lerner J, Goldfarb M. Follicular variant papillary thyroid carcinoma in a pediatric population. Pediatr Blood Cancer 2015;62:1942-1946. Epub 2015 Jul 1

35. Welch Dinauer CA, Tuttle RM, Robie DK, McClellan DR, Svec RL, Adair C, Francis GL. Clinical features associated with metastasis and 
recurrence of differentiated thyroid cancer in children, adolescents and young adults. Clin Endocrinol (Oxf) 1998;49:619-628.

36. Hung W, Sarlis NJ. Current controversies in the management of pediatric patients with well-differentiated nonmedullarythyroid cancer: A review. Thyroid 2002;12:683-702.

37. Demidchik YE, Demidchik EP, Reiners C, Biko J, Mine M, Saenko VA, Yamashita S. Comprehensive clinical assessment of 740 cases of surgically treated thyroid cancer in children of Belarus. Ann Surg 2006:243:525-532.
38. Handkiewicz-Junak D, Wloch J, Roskosz J, Krajewska J, Kropinska A, Pomorski L, Kukulska A, Prokurat A, Wygoda Z, Jarzab B. Total thyroidectomy and adjuvant radioiodine treatment independently decrease locoregional recurrence risk in childhood and adolescent differentiated thyroid cancer. J Nucl Med 2007;48:879-888.

39. O'Gorman CS, Hamilton J, Rachmiel M, Gupta A, Ngan BY, Daneman D. Thyroid cancer in childhood: A retrospective review of childhood course. Thyroid 2010;20:375-380. 